\title{
Variáveis sociodemográficas como determinantes do domínio meio ambiente da qualidade de vida de adolescentes
}

\author{
Sociodemographic variables as determinant \\ of the environment domain of quality of life of adolescents
}

\author{
Alex Pinheiro Gordia ${ }^{1,2}$ \\ Teresa M aria Bianchini de Q uadros ${ }^{2}$ \\ Wagner de Campos ${ }^{1}$
}

${ }^{1}$ Centro de Pesquisa em Exercício e Esporte, Departamento deEducação Física, Universidade Federal do Paraná. Rua Coração de M aria 92. 80215-370 Curitiba PR.

alexgordia@gmail.com

${ }^{2} \mathrm{~N}$ úcleo de Pesquisa em Cineantropometriae

Desempenho Humano, UniversidadeFederal de Santa Catarina.
Abstract This study aimed to identify the relationship between sociodemographic variables and environment domain of Quality of Life ( $Q O L)$ of adolescents. The sample was composed by 608 adolescents aged 14 to 20 years, from the municipality of Lapa, Paraná State, South of Brazil. The WHOQOL-Bref and ABEP questionnaires were used, respectively, to assess the $\mathrm{Q} \mathrm{OL}$ and socioeconomic condition. Data analysis used descriptive statistics and chi-square tests, exact Fisher and logistic regression binary, considering $p<0.05$. The average value of $\mathrm{QOL}$ overall and environment domain of adolescents was $67.3 \%$ and $55.6 \%$, respectively. Girlswere 1.9 times morelikely to present poor environment domain than boys, and less wealthy individuals ( $B$ and $C+D+E$ classes) showed, respectively, 5.7 and 7.4 times more risk to have poor environment domain when compared with adolescents of $A$ class socioeconomic. It can be inferred that the environment domain pre sented themselves as vulnerable point of the $\mathrm{QO} \mathrm{L}$ of adolescents investigated. The main groups in risk to the have poor environment domain of the Q O L werecomposed by femaleadolescents, belonging to less advantaged socioeconomic classes. Key words Environment, WHOQ OL-Bref, Adolescents, Public policy, Socioeconomic condition, Sociodemographic variables
Resumo 0 presente estudo teve como objetivo identificar a relação entre variáveis sociodemográficas e o domínio meio ambiente da qualidade de vida (QV) de adolescentes. A amostra foi composta por 608 adolescentes com idades entre 14 e20 anos, do município da Lapa (PR). Para a avaliação da QV e da condição socioeconômica, foram utilizados, respectivamente, o questionário W H OQOL-Bref e da ABEP. Para análise dos dados, re correu-se à estatística descritiva e aos testes quiquadrado, exato de Fisher e regressão logística binária, considerando $p<0,05.0$ valor médio da QV global e do domínio meio ambiente dos adolescentes foi $67,3 \%$ e $55,6 \%$, respectivamente. M oças tiveram 1,9 vezes mais chance para apresentar domínio meio ambiente ruim do que rapazes, bem como indivíduos menos abastados (classe $B$ e $C+D+E$ ) apresentaram, respectivamente, 5,7 e 7,4 vezes mais risco para possuir domínio meio ambiente ruim quando comparados com adolescentes da classe socioeconômica A. Pode-se inferir que o domínio meio ambiente apresentou-se como ponto vulnerável da QV dosadolescentes investigados. Os principais subgrupos em risco para possuir domínio meio ambiente da QV ruim foram compostos por adolescentes do sexo feminino e pertencentes às classes socioeconômicas menos favorecidas. Palavras-chave M eio ambiente, WHOQOL-Bref, Adolescentes, Políticas públicas, Condição socioe conômica, Variáveis sociodemográficas 
Introdução

Nas últimas décadas, o interesse crescente da comunidade científica e de todo o setor de saúde em relação à qualidade de vida (QV) levou a um desenvolvimento marcante desse construto ${ }^{1}$. Neste sentido, diversos instrumentos têm sido desenvolvidos visando mensurar, de forma confiável, a QV de diferentes populações. Dentre esses instrumentos, o WHOQOL-Bref, desenvolvido pelo grupo de estudos sobre QV da Organização M undial da Saúde'2, tem apresentado alta aceitação, sendo testado e validado em diversos países, inclusive no Brasili3,4. Com o WHOQ OLBref, pode-se inferir sobre a QV global e domínios físico, psicológico, das relações sociais e do meio ambiente, possibilitando a identificação das principais demandas da população estudada de forma pontual, visando à elaboração de políticas públicas de promoção da saúde.

0 domínio meio ambiente do questionário WH OQ OL-Bref refere-se às facetas de segurança física e proteção, ambiente no lar, recursos financeiros, disponibilidade e qualidade dos cuidados de saúde e sociais, oportunidade de adquirir novas informações e habilidades, participação e oportunidades de recreação/lazer e ambiente físico (poluição, ruído, trânsito, clima e transporte). Pesquisas destacam que fatores ambientais, como o saneamento básico, segurança pública, cuidados desaúde esociais, poluição, trânsito, transporte e clima interferem ne gativamente na QV da população brasileira ${ }^{5-9}$. No entanto, poucos estudos são realizados com adolescentes, especialmente utilizando o WHOQOL-Bref, para mensurar a QV.

Há indícios de que aspectos sociodemográficos estão associados a condições ambientais de diferentes populações ${ }^{10-12}$. Todavia, investigações que avaliem variáveis determinantes de fatores ambientais diretamente relacionados à QV ainda são incipientes. Desta forma, o objetivo do presenteestudo foi avaliar o domínio meio ambiente da QV de adolescentes, bem como investigar sua relação com variáveis sociodemográficas.

\section{Métodos}

População e amostra

0 presente estudo foi desenvolvido com base no banco de dados do projeto de pesquisa "Análise da qualidade de vida, prevalência do consumo deálcool, nível de atividade física eíndice de massa corporal em estudantes do ensino médio da cidade da Lapa, PR", realizado de junho a novembro de 2005. Os protocolos do estudo foram aprovados pelo Comitê de Ética em Pesquisa da Universidade Estadual de Ponta Grossa, Paraná, Brasil, Processo n 020/2005.

0 projeto envolveu estudantes de 14 a 20 anos de idade, de ambos os sexos, regularmente matriculados na rede pública e particular do ensino médio do município da Lapa, integrante da região metropolitana de Curitiba, capital do Estado do Paraná, Região Sul do Brasil. A população do município no ano de 2005 foi estimada em 44.733 habitantes $^{13}$. A rededeensino contava com 1.596 estudantes regularmente matriculados do 1o ao 3 o ano do ensino médio, distribuídos em sete escolas: duas da rede particular ( $N=75)$; três públicas de área rural $(\mathrm{N}=338)$ e duas públicas de área urbana ( $N=1183)$, segundo informação do N úcleo Municipal deEducação. A perspectiva do estudo foi avaliar todos os adolescentes matriculados no ensino médio. No entanto, a amostra final incluiu 608 sujeitos entre 14 e 20 anos de idade, que tiveram todos os dados coletados e queparticiparam voluntariamente, representando $38,1 \%$ da população de estudantes do ensino médio, subdivididos nos seguintes estratos: escolas particulares, 24 adolescentes (representando $32 \%$ desse estrato); escolas públicas da área urbana, 391 adolescentes (33,1\% do estrato); escolas públicas da área rural, 193 adolescentes (57,1\% do estrato). Visando confirmar a representatividade da amostra investigada em relação à população de estudo, foi calculado o erro amostral de acordo com os critérios estabelecidos por $\mathrm{Gi}^{14}$, que aponta um erro inferior a $1 \%$ como garantia da representatividade da amostra. No presente estudo, foi encontrado o erro amostral de $0,7 \%$. Os dados de caracterização da amostra estudada podem ser observados na Tabela 1.

\section{Instrumentos e procedimentos}

Primeiramente, foi enviado aos pais e/ ou responsáveis dos adolescentes o termo de consentimento livree esclarecido (TCLE). Com a devolução do TCLE devidamente assinado, o adolescente era incluído na amostra para a subsequente avaliação. Foram investigadas variáveis referentes à QV dos adolescentes (domínio meio ambiente), massa corporal, estatura e variáveis sociodemográficas (sexo, idade, condição socioeconômica, tipo de escola, local demoradia, série escolar e turno de estudo). 


\begin{tabular}{|c|c|c|c|c|}
\hline & $\mathrm{n}$ & Idade (anos) & Massa corporal (kg) & Estatura (m) \\
\hline \multicolumn{5}{|l|}{ Sexo } \\
\hline M asculino & 239 & $16,01(1,33)$ & $62,69(11,04)$ & $1,73(0,08)$ \\
\hline Feminino & 369 & $15,76(1,18)$ & $55,65(8,92)$ & $1,62(0,07)$ \\
\hline \multicolumn{5}{|c|}{ Condição socioeconômica } \\
\hline A & 31 & $15,74(1,26)$ & $67,15(17,37)$ & $1,70(0,09)$ \\
\hline B & 163 & $15,74(1,12)$ & $58,37(10,23)$ & $1,67(0,09)$ \\
\hline C & 279 & $15,93(1,26)$ & $58,30(9,45)$ & $1,66(0,09)$ \\
\hline D & 128 & $15,92(1,37)$ & $56,79(9,42)$ & $1,64(0,08)$ \\
\hline $\mathrm{E}$ & 7 & $15,00(0,82)$ & $55,50(9,47)$ & $1,61(0,08)$ \\
\hline \multicolumn{5}{|l|}{ Tipo de escola } \\
\hline Pública & 584 & $15,88(1,25)$ & $58,23(10,06)$ & $1,66(0,09)$ \\
\hline Particular & 24 & $15,21(0,93)$ & $63,06(15,92)$ & $1,67(0,08)$ \\
\hline \multicolumn{5}{|c|}{ Local de moradia } \\
\hline Área urbana & 415 & $15,87(1,21)$ & $58,18(10,72)$ & $1,66(0,09)$ \\
\hline Área rural & 193 & $15,82(1,32)$ & $58,93(9,63)$ & $1,67(0,09)$ \\
\hline \multicolumn{5}{|l|}{ Série escolar } \\
\hline $1^{\circ}$ ano & 256 & $15,07(1,01)$ & $56,55(9,58)$ & $1,65(0,09)$ \\
\hline $2^{\circ}$ ano & 219 & $16,12(1,09)$ & $59,52(9,76)$ & $1,67(0,09)$ \\
\hline $3^{\circ}$ ano & 133 & $16,93(0,84)$ & $60,20(12,21)$ & $1,68(0,09)$ \\
\hline \multicolumn{5}{|l|}{ Turno de estudo } \\
\hline M atutino & 302 & $15,74(1,21)$ & $59,44(10,79)$ & $1,67(0,09)$ \\
\hline Vespertino & 203 & $15,82(1,22)$ & $56,82(9,53)$ & $1,65(0,08)$ \\
\hline Noturno & 103 & $16,27(1,32)$ & $58,57(10,48)$ & $1,66(0,10)$ \\
\hline Amostra total & 608 & $15,86(1,24)$ & $58,42(10,38)$ & $1,66(0,09)$ \\
\hline
\end{tabular}

A QV foi mensurada com o uso do questionário WHOQOL-Bref, que contém 26 questões. Ao preencher o instrumento, o participante deve considerar os últimos quinze dias vividos. I zutsu et $\mathrm{al}^{15}$ demonstraram que o WHOQOL-Bref possui um conteúdo válido epropriedades psicométricas aceitáveis para mensurar a QV de adolescentes. Para o presente estudo, foram utilizadas apenas as informações referentes ao domínio meio ambiente. Estedomínio écomposto por oito questões, a saber: 1) Quão seguro (a) vocêsesente em sua vida diária?; 2) Quão saudável é o seu ambientefísico (clima, barulho, poluição, atrativos)?; 3) Você tem dinheiro suficiente para satisfazer suas necessidades?; 4) Quão disponíveis para você estão as informações que precisa no seu dia a dia?; 5) Em que medida você tem oportunidades de atividade de lazer?; 6) Quão satisfeito (a) você está com as condições do local onde mora?; 7) Quão satisfeito (a) você está com o seu acesso aos serviços de saúde?; 8) Q uão satisfeito (a) você está com o seu meio de transporte?

O WHOQOL-Bref foi analisado com base nos critérios propostos pela equipe australiana do WHOQ OL ${ }^{16}$, os quais permitem a classificação da QV em uma escala percentual de 0 a 100.
Quanto mais próximo de $100 \%$, melhor é a QV do avaliado.

Foram coletados dados de massa corporal (MC, kg), com uma balança digital Plenna, com capacidade para $150 \mathrm{~kg}$ e resolução de $100 \mathrm{~g}$, estando o avaliado descalço e com o mínimo de roupa possível. A estatura (EST, $\mathrm{Cm}$ ) foi aferida utilizando-se uma fita métrica, com escal as graduadas de 0,1cm, fixada em uma superfície de apoio plana, sendo que para efetuar a medida 0 avaliado deveria estar descalço ou no máximo de meias, em apnéia inspiratória e com a cabeça posicionada no plano de Frankfurt ${ }^{17}$.

A condição socioeconômica foi avaliada de acordo com o Critério de Classificação Econômica do Brasil (CCEB), desenvolvido pela Associação Brasileira deEmpresas de Pesquisa (ABEP) ${ }^{18}$. O CCEB estima o poder de compra das pessoas e das famílias e o grau de instrução do chefe da família, classificando da seguinte forma: classe $A$ ( 25 a 34 pontos), classe $B$ ( 17 a 24 pontos), classe C (11 a 16 pontos), classe D (6 a 10 pontos) e classeE ( 0 a 5 pontos). Para esteestudo, as classes socioeconômicas foram separadas em classe alta $(A)$, classe média (B) e classe baixa $(C+D+E)$, visando ao poder das análises estatísticas. 
Análise estatística

Inicialmente, recorreu-se à análise descritiva da QV global e do domínio meio ambiente dos adolescentes estudados. Em seguida, utilizou-seo teste do qui-quadrado e, quando necessário, 0 teste exato de Fisher, para investigar diferenças proporcionais referentes ao domínio meio ambienteentre os seguintes grupos: sexo, idade, condição socioeconômica, tipo de escola, local de moradia, série escolar e turno de estudo. A regressão logística binária (análise bruta eajustada) foi usada para examinar as associações entre os fatores sociodemográficos (variáveisindependentes) com o domínio meio ambiente (variável dependente) dos adolescentes. Tanto para o qui-quadrado quanto para regressão, a percepção do domínio meio ambiente da QV dos adolescentes foi dicotomizada em positiva e negativa com base na mediana. Para modelagem da análise de regressão, foram consideradas apenas as variáveis que apresentaram nível designificância inferior a 0,20 para associação com o domínio psicológico no testederazões deverossimilhança. 0 nível de significância foi estabelecido em $p<0,05$.

\section{Resultados}

Com base na análise do questionário WHOQOL-Bref, observou-se valor médio da QV global dos adolescentes (média de todos os domínios) de $67,3 \%$, com desvio padrão de $10,2 \%$. Para o domínio meio ambiente, foi encontrado valor médio de $55,6 \%$, com desvio padrão de $11,4 \%$. As análises referentes às proporções para o domínio meio ambiente da QV, com estratificação por variáveis sociodemográficas, podem ser observadas na Tabela 2. Os achados demonstraram que o sexo, a condição socioeconômica e 0 tipo de escola estiveram associados ao domínio meio ambiente.

\begin{tabular}{|c|c|c|c|}
\hline \multirow{2}{*}{$\begin{array}{c}\text { Variáveis } \\
\text { sociodemográficas }\end{array}$} & \multicolumn{2}{|c|}{ Percepção do domínio meio ambiente (\%) } & \multirow[b]{2}{*}{$\mathrm{p}$} \\
\hline & Positiva & Negativa & \\
\hline \multicolumn{4}{|l|}{ Sexo } \\
\hline Masculino & 62,3 & 37,7 & \multirow[t]{2}{*}{0,001} \\
\hline Feminino & 47,4 & 52,6 & \\
\hline \multicolumn{4}{|l|}{ Idade (anos) } \\
\hline 14 & 52,1 & 47,9 & \multirow{7}{*}{0,845} \\
\hline 15 & 55,9 & 44,1 & \\
\hline 16 & 53,5 & 46,5 & \\
\hline 17 & 54,5 & 45,5 & \\
\hline 18 & 43,2 & 56,8 & \\
\hline 19 & 45,5 & 54,5 & \\
\hline 20 & 50,0 & 50,0 & \\
\hline \multicolumn{4}{|l|}{ Condição socioeconômica } \\
\hline A & 90,3 & 9,7 & \multirow{3}{*}{0,001} \\
\hline B & 56,4 & 43,6 & \\
\hline$C, D$ e E & 49,3 & 50,7 & \\
\hline \multicolumn{4}{|l|}{ Tipo de escola } \\
\hline Pública & 87,5 & 12,5 & \multirow{2}{*}{0,001} \\
\hline Particular & 51,9 & 48,1 & \\
\hline \multicolumn{4}{|l|}{ Local de moradia } \\
\hline Área rural & 52,3 & 47,7 & \multirow[t]{2}{*}{0,747} \\
\hline Área urbana & 53,7 & 46,3 & \\
\hline \multicolumn{4}{|l|}{ Série escolar } \\
\hline 1o ano & 50,4 & 49,6 & \multirow[t]{3}{*}{0,473} \\
\hline $2^{\circ}$ ano & 55,3 & 44,7 & \\
\hline 3o ano & 55,6 & 44,4 & \\
\hline \multicolumn{4}{|l|}{ Turno de estudo } \\
\hline M atutino & 55,3 & 44,7 & \multirow{3}{*}{0,101} \\
\hline Vespertino & 55,2 & 44,8 & \\
\hline Noturno & 43,7 & 56,3 & \\
\hline
\end{tabular}


Os resultados referentes à análise de regressão logística bruta demonstraram associação entre o domínio meio ambientecom o sexo, condição socioeconômica, tipo de escola e turno de estudo. Quando considerada a análise ajustada, permaneceram associados ao domínio meio ambienteo sexo ea condição socioeconômica (Tabela 3). Adolescentes do sexo feminino tiveram 1,9 vezes mais chance para apresentar percep ção negativa do domínio meio ambiente do que seus pares do sexo masculino ( $R C=1,96 ;$ IC 95\% 1,39-2,77; $p<0,001$ ). Indivíduos menos abastados (classe $\mathrm{B}$ eC $+\mathrm{D}+\mathrm{E}$ ) apresentaram, respectivamente, 5,7e 7,4 vezes mais risco para possuir percepção negativa do domínio meio ambiente quando comparados com adolescentes da classe socioeconômica $A$ ( $R C=5,68$; IC $95 \%$ 1,58-20,42; $p=0,008$ e RC=7,41; IC 95\% $2,08-26,38 ; p=0,002$, respectivamente).

\section{Discussão}

Os achados do presente estudo indicaram o meio ambiente como domínio vulnerável da QV dos adolescentes estudados, com valor médio de $55,6 \%$. Estes resultados são preocupantes e evidenciam a necessidade de investimentos governamentais visando à mel horia das condições ambientais desta população, principalmente em rela- ção à segurança física e proteção, ambiente no lar, recursos financeiros, cuidados de saúde e sociais, oportunidade de adquirir novas informações e habilidades, participação e oportunidades de recreação/lazer, ambiente físico, poluição, ruído, trânsito, clima e transporte'. Este estudo foi pioneiro na avaliação do domínio meio ambiente da QV de adolescentes brasileiros, bem como apresentou resultados relevantes em relação às variáveis sociodemográficas relacionadas a este domínio. A escassez de pesquisas referentes a aspectos ambientais diretamente relacionados à QV de adolescentes, principalmenteutilizando o instrumento WHOQOL-Bref, dificulta a comparação dos achados obtidos no presente estudo.

A maioria dos estudos nacionais que utilizaram o WHOQOL tem demonstrado que o domínio meio ambiente é a pior faceta da QV da população brasileira. Gordia, Quadros e Vilela Júnior ${ }^{5}$ analisaram a QV de indivíduos do exército brasileiro e observaram os menores valores percentuais para este domínio, sendo, portanto, o principal ponto vulnerável na QV deste segmento da população. 0 mesmo pode ser observado em outras amostras; Gordia et al. ${ }^{6}$ avaliaram a QV de mulheres idosas da cidade de Fortaleza (CE) e os achados indicaram índices preocupantes para questões ambientais da QV. Penteado e Pereira ${ }^{7}$ avaliaram a QV de 128 professo-

Tabela 3. Associação entre o domínio meio ambiente da QV com variáveis sociodemográficas em adolescentes.

\begin{tabular}{|c|c|c|c|}
\hline Variáveis (Categorias) & $\begin{array}{c}\text { Percepção negativa } \\
\text { do DMA } A^{\S}(\%)\end{array}$ & $\begin{array}{l}\text { Análise bruta } \\
\text { RC }(95 \% \text { IC) }\end{array}$ & $\begin{array}{c}\text { Análise ajustada } \\
\text { RC }(95 \% \text { IC) }\end{array}$ \\
\hline \multicolumn{4}{|l|}{ Sexo } \\
\hline M asculino & 37,7 & $1 *$ & $1^{\dagger}$ \\
\hline Feminino & 52,6 & $1,84(1,32-2,56)$ & $1,96(1,39-2,77)$ \\
\hline \multicolumn{4}{|l|}{ Condição socioeconômica } \\
\hline$A$ & 9,7 & $1^{*}$ & $1^{\dagger}$ \\
\hline B & 43,6 & $7,20(2,11-24,65)$ & $5,68(1,58-20,42)$ \\
\hline$C, D$ e $E$ & 50,7 & $9,61(2,88-32,10)$ & $7,41(2,08-26,38)$ \\
\hline \multicolumn{4}{|l|}{ Tipo de escola } \\
\hline Particular & 12,5 & $1 *$ & 1 \\
\hline Pública & 48,1 & $6,49(1,92-22,00)$ & $3,04(0,81-11,47)$ \\
\hline \multicolumn{4}{|l|}{ Turno de estudo } \\
\hline M atutino & 44,7 & $1^{*}$ & 1 \\
\hline Vespertino & 44,8 & $1,01(0,70-1,44)$ & $0,77(0,52-1,12)$ \\
\hline Noturno & 56,3 & $1,59(1,02-2,50)$ & $1,51(0,95-2,43)$ \\
\hline
\end{tabular}

Nota:

$* p<0,05$ para a análise bruta.

† $p<0,05$ para a análise ajustada.

¥Análise ajustada para as demais variáveis independentes do modelo de regressão.

${ }^{\S} \mathrm{DM} A=$ Domínio M eio Ambiente. 
res de ensino médio de quatro escolas estaduais de Rio Claro (SP) e observaram que o domínio meio ambiente foi a faceta mais preocupante da QV dessa amostra. Em investigação real izada por Castro et al. ${ }^{9}$, com 276 adultos dependentes de tabaco, observou-se que o meio ambiente foi 0 principal aspecto negativo da QV.

Considerando os problemas urbanos contemporâneos e as possibilidades que a cidade oferece para a realização de projetos sociais, a Organização Mundial da Saúde (OM S) e suas agências regionais, como a Organização PanAmericana da Saúde (OPAS), iniciaram o M ovimento Cidades Saudáveis, com o intuito de motivar governos e sociedade civil a desenvolver estratégias, em diversos setores das políticas sociais, com a implementação de projetos interinstitucionais e intersetoriais, visando realizar ações de melhoria das condições de vida, saúdee, consequentemente, da QV da população urbana ${ }^{19}$.

Recentemente, alguns municípios brasileiros - por exemplo, Curitiba (PR), Chopinzinho (PR) e Vinhedo (SP) -, preocupados com as condições de vida da população, estão implementando programas de intervenção para melhoria de diversos fatores, tais como saneamento básico, educação, assistência médica, ambientes de trabalho e lazer saudável ${ }^{20}$. Entretanto, o comprometimento dos governantes com a melhoria das condições de vida dos brasileiros ainda é muito reduzido eeste descaso está explícito nos resultados obtidos no presente estudo.

Os achados da regressão logística ajustada indicaram o sexo e a condição socioeconômica como variáveis determinantes do domínio meio ambiente da QV dos adolescentes investigados. Moças apresentaram maior risco para possuir percepção negativa do domínio meio ambiente do que rapazes. Este fato pode estar relacionado a diferenças nas condições de vida ou a diferenças na percepção da QV. No entanto, tendo em vista que outros estudos, realizados com jovens e adultos, também vêm observando o sexo feminino como grupo de risco para apresentar percepção negativa da QV ${ }^{12,21,22}$, pode-se supor que a principal diferença na avaliação do domínio meio ambiente da QV entre rapazes e moças esteja na maior exigência das adolescentes do sexo feminino em relação à percepção da QV, ou seja, rapazes e moças podem ter condições de vida semelhantes, porém, formas diferentes para analisar e ponderar diversos aspectos de sua vida. Entretanto, considerando que o presente estudo teve um design transversal, este resultado deve ser analisado com cautela, pois não é possível estabelecer uma relação de causa e efeito entre 0 sexo e o domínio meio ambiente da Q V dosadolescentes. Desta forma, outros estudos são necessários para confirmar estes achados, em especial, pesquisas com enfoquequalitativo referente à forma como os adolescentes percebem e avaliam sua QV.

A variável queapresentou relação mais consistente com o domínio meio ambientefoi a condição socioeconômica, tendo em vista que indivíduos menos abastados (classe B e C $+D+E$ ) apresentaram, respectivamente, 5,7 e 7,4 vezes mais risco para possuir percepção negativa do domínio meio ambiente quando comparados com adolescentes da classesocioeconômica A. Considerando pesquisas com design diferente do presente estudo, porém com o mesmo fim, parece que indivíduos pertencentes à classes menos favorecidas convivem com condições ambientais desfavoráveis e prejudiciais à sua QV 10-12. Szwarcwald et al. ${ }^{10}$ testaram a associação entre desigualdade de renda e condições de saúde no município do Rio de aneiro. Os resultados evidenciam correlações significativas dos indicadores de desigual dade de renda com todos os indicadores de saúde, demonstrando que as piores condições de saúde não podem ser dissociadas das disparidades de renda. Para os homicídios, a concentração de indivíduos residentes em favelas se mostrou relevante, sugerindo uma piora adicional das condições de saúde através da deterioração das interações comunitárias e do aumento da criminalidade. Uma análise geoepidemiológica apontou para o vínculo entre as piores condições de saúde e a concentração residencial de pobreza. Os autores concluíram que há necessidade urgente de se implementarem políticas compensatórias para amenizar os efeitos danosos da desigual dade social.

Kran e Ferreira ${ }^{11}$ buscaram discutir a QV na cidade de Palmas, capital do Estado do Tocantins, através de uma análise da habitação e seu entorno. Os autores observaram que Palmas possui diversas áreas periféricas com acessibilidade e infraestrutura limitadas, onde o solo é menos valorizado e ocupado, basicamente, por populações de baixa renda, que vivem em evidentes condições de precariedade econômica, social e ambiental, tendo, por consequência, a sua QV comprometida. Pesquisa desenvolvida por Gaspar et al. ${ }^{12}$ objetivou investigar a QV relacionada à saúde em função da condição socioeconômica. Osachados do estudo indicaram que adolescentes com condição socioeconômica mé dia/alta apresentaram valores médios estatisticamente mais elevados para variáveis socioam- 
bientais da QV do que seus pares de condição socioeconômica baixa.

De uma forma geral, adolescentes de condição socioeconômica menos privilegiada apresentam maior chance para morar em regiões perifé ricas, sem saneamento básico, vivem em áreas com alto índice de criminalidade, convivem com um sistema educacional e de saúde falido, não têm oportunidades para lazer, apresentam dificuldades financeiras, al imentação restrita e acesso limitado a novas tecnologias. É evidente que estes fatores implicam a diminuição da QV desta população, em especial sobre aspectos relacionados ao meio ambiente, fato confirmado pelo presente estudo e demais investigações supracitadas. N este contexto, torna-se patente a necessidade de interven ção governamental através de políticas públicas eficientes que visem melhorar as condições de vida da população que apresenta dificuldades socioeconômicas, especial menteem relação a aspectos-chave, como sistema educacinal, de saúde e de segurança pública.

\section{Conclusão}

O domínio meio ambiente apresentou-se como ponto vulnerável da QV dos adolescentes investigados, indicando a urgência para implementação de políticas públicas ambientais visando melhorar a QV desta população. Os principais subgrupos em risco para apresentar percepção negativa do domínio meio ambiente da QV foram compostos por adolescentes do sexo feminino e pertencentes às classes socioeconômicas menos favorecidas.

\section{Colaboradores}

AP Gordia participou da concepção, delineamento e redação do artigo, análise e interpretação de dados. TM B de Quadros participou da concepção, delineamento e redação do artigo, análise e interpretação de dados. W de Campos participou da concepção, delineamento, redação do artigo e revisão crítica. 


\section{Referências}

1. Campolina AG, Ciconelli RM . Qualidade de vida e medidas de utilidade: parâmetros clínicos para as tomadas de decisão em saúde. Rev. Panam. Salud Públ. / Pan Am. J. Public H ealth 2006; 19(2):128-136.

2. The Whoqol Group. The World Health Organization quality of life assessment (WHOQOL): development and general psychometric properties. Soc Sci M ed 1998; 46:1569-1585.

3. Fleck M PA, Louzada S, Xavier M, Chachamovich E, Vieira G, Santos L, Pinzon V. Aplicação da versão em português do instrumento abreviado de avaliação da qualidade de vida "WHOQOL-bref". Rev. Saude Publica 2000; 34(2):178-183.

4. Moreno $A B$, Faerstein $E$, Werneck $G L$, Lopes CS Chor D. Propriedades psicométricas do Instrumento Abreviado de Avaliação de Qualidade de Vida da Organização M undial da Saúde no Estudo Pró-Saúde. Cad Saude Publica 2006; 22(12):2585-2597.

5. Gordia AP, Quadros TM B, Vilela Júnior GB. Quality of life and physical fitness of individuals in the Brazilian army. The FIEP bulletin 2006; 76(Special Edition):82-85.

6. Gordia AP, Quadros TMB, Vilela Júnior GB, Souza EA, Cabral C, M orais TB, Quadros Junior PK, Campos W. Comparação da qualidade de vida de muIheres idosas praticantes e não praticantes de exercício físico. Lecturas: Educación Física y Deportes 2007; 11(106):1-2.

7. Penteado RZ, Pereira IMTB. Qualidade de vida e saúde vocal de professores. Rev. Saude Publica 2007; 41(2):236-243.

8. Siviero LMPS. Saúde mental e qualidade de vida de infartados [tese]. Ribeirão Preto (SP): Escola de Enfermagem de Ribeirão Preto; 2003.

9. Castro MG, Oliveira MS, Moraes JFD, Miguel AC, Araujo RB. Qualidade de vida e gravidade da dependência de tabaco. Rev Psiquiatr Clín 2007; $34(2): 61-67$.

10. Szwarcwald CL, Bastos FI, Esteves MAP, Andrade CLT, Paez MS, Medici EV, Derrico M. Desigualdade de renda e situação de saúde: o caso do Rio de Janeiro. Cad Saude Publica 1999; 15(1):15-28.

11. Kran F, Ferreira FPM . Qualidade de vida na cidade de Palmas - TO: uma análise através de indicadores habitacionais e ambientais urbanos. Ambient Soc 2006; 9(2):123-141.

12. Gaspar T, M atos M G, Ribeiro JLP, Leal I. Qualidade de vida e bem-estar em crianças e adolescentes. Rev Bras Ter Cogn 2006; 2(2):47-60.
13. Instituto Brasileiro de Geografia e Estatística IBGE. Projeção da População do Brasil: 1980-2050. [acessado 2005 mar 12]. Disponível em: http://www. ibge.gov.br/home/estatistica/populacao/projecao_ da_populacao/default.shtm

14. Gil AC. M étodos e técnicas de pesquisa social. 3a ed. São Paulo: Atlas; 1991

15. Izutsu T, Tsutsumi A, Islam A, Matsuo Y, Yamada $\mathrm{HS}$, Kurita H, Wakai S. Validity and reliability of the Bangla version of WHOQOLBREF on an adolescent population in Bangladesh. Qual Life Res 2005; 14(7):1783-1789.

16. The Australian Centre for Posttraumatic M ental $\mathrm{H}$ ealth. Trauma related research, training and policy development, 2003. [acessado 2006 abr 15]. Disponível em: http://www.acpmh.unimelb.edu.au/whoqol/ whogol-bref_5.html

17. Gordon CC, Chumlea WCC, Roche AF. Stature, recumbent length, and weight. In: Lohman TG, Roche AF, M artorell R, editors. Anthropometric standardization reference manual. Champaign: Human Kinetics; 1988.

18. Associação Brasileira de Empresas de Pesquisa. Dados com base no Levantamento Sócio Econômico, 2000 - IBOPE. [acessado 2005 mai 10]. Disponível em: http://www.abep.org

19. Westphal MF. O Movimento Cidades/Municípios Saudáveis: um compromisso com a qualidade de vida. Cien Saude Colet 2000; 5(1):39-51.

20. Vilarta R. Qualidade de vida e políticas públicas: saúde, lazer e atividade física. Campinas: IPES Editorial; 2004.

21. Lipp MEN, Tanganelli MS. Stress e qualidade de vida em Magistrados da Justiça do Trabalho: diferenças entre homens e mulheres. Psicol Refl Crít 2002; 15(3):537-548.

22. Cucchiaro G, Dalgalarrondo P. Saúde mental e qualidade de vida em adolescentes: um estudo entre escolares em duas áreas urbanas contrastantes. Rev Bras Psiquiatr 2007; 29(3):213-221.

Artigo apresentado em 21/07/08

Aprovado em 09/10/08

Versão final apresentada em 29/10/08 